Int. J. Contemp. Math. Sci., Vol. 1, 2006, no. 2, 67 - 79

\title{
On a New Class of p-Valent Functions with Negative Coefficients
}

\author{
H. Özlem Güney and S. Sümer Eker \\ University of Dicle, Faculty of Science and Art \\ Department of Mathematics \\ 21280, Diyarbakır, Turkey \\ ozlemg@dicle.edu.tr \& sevtaps@dicle.edu.tr
}

\begin{abstract}
In this paper, we introduce the class $A_{k}^{*}(p, A, B, \alpha)$ of p-valent functions in the unit disc $\mathbb{U}=\{z \in \mathbb{C}:|z|<1\}$. We give basic properties of functions belonging to this class and obtain numerous sharp results including coefficient estimate, distortion theorems, closure theorems, integral operators and linear combinations of several functions belonging to this class. We also obtain radii of close-to-convexity, starlikeness and convexity for functions belonging to $A_{k}^{*}(p, A, B, \alpha)$. Furthermore, various distortion inequalities for fractional calculus of functions in this class are given.
\end{abstract}

Mathematics Subject Classification: Primary 30C45, Secondary 30C50

Keywords: p-valent function, distortion, coefficient, closure,close-to-convex, starlike, fractional calculus, convex.

\section{Introduction}

Let $\mathcal{A}_{(p)}(p \geq 2)$ denote the class of functions $f(z)$ of the form

$$
f(z)=z^{p}+\sum_{n=1}^{\infty} a_{p+n} z^{p+n}
$$

which are analytic and p-valent in the open unit disk $\mathbb{U}=\{z \in \mathbb{C}:|z|<1\}$. A function $f$ belonging to the class $\mathcal{A}_{(p)}$ is said to be in the class $A_{(p)}^{*}(p, A, B, \alpha)$ 
if and only if

$$
\operatorname{Re}\left\{\frac{z f^{(p-1)(z)}}{f^{(p-2)(z)}}\right\}>\frac{\alpha}{p}
$$

for $-1 \leq B<A \leq 1,-1 \leq B<0$ and $0 \leq \alpha<p$ and all $z \in \mathbb{U}$.

In the other words, $f \in A_{(p)}^{*}(p, A, B, \alpha)$ if and only if if there exists a function $w$ satisfying $w(0)=0$ and $|w(z)|<1$ for $z \in \mathbb{U}$ such that

$$
\frac{z f^{(p-1)(z)}}{f^{(p-2)(z)}}=\left(2-\frac{\alpha}{p}\right) \frac{1+A w(z)}{1+B w(z)}+\frac{\alpha}{p} .
$$

The condition (1.2) is equivalent to

$$
\left|\frac{p \frac{f^{(p-1)}(z)}{f^{(p-2)}(z)}-2 p}{(A-B)(2 p-\alpha)+2 p B-p B \frac{f^{(p-1)}(z)}{f^{(p-2)}(z)}}\right|<1, \quad z \in \mathbb{U} .
$$

Let $A_{k}$ denote the subclass of $A_{(p)}$ consisting of functions analytic and p-valent which can be expressed in the form

$$
f(z)=z^{p}-\sum_{n=1}^{\infty} a_{p+n} z^{p+n}
$$

where $a_{p+n}>0$.

Let us define $A_{k}^{*}(p, A, B, \alpha)=A_{(p)}^{*}(p, A, B, \alpha) \cap A_{k}$.

M.K.Aouf and H.E.Darwish[4], S.L.Shulka and Dastrath [5] have studied certain classes of analytic functions with negative coefficients. Also, the class $A_{(p)}$ is studied by M.Nunokawa and S.Hoshino [3].

In this paper,we obtain coefficient estimates, distortion theorems, covering theorem, integral operators, radii of close-to-convexity, starlikeness and convexity for functions belonging to $A_{k}^{*}(p, A, B, \alpha)$. Further, it is shown that this class is closed under "arithmetic mean" and "convex linear combinations". Also, various distortion theorems for fractional calculus of functions are given.

\section{Coefficient Estimates}

Theorem 1 Let the function $f$ be defined by (1.4). Then $f \in A_{k}^{*}(p, A, B, \alpha)$ if and only if

$$
\sum_{n=1}^{\infty} \frac{[p n(1-B)+(A-B)(2 p-\alpha)](p+n) !}{(n+2) !} a_{p+n} \leq \frac{1}{2}(A-B)(2 p-\alpha) p !
$$


The result is sharp.

Proof Assume that the inequality (2.1) holds true and let $|z|=1$. Then we obtain

$$
\begin{aligned}
& \left|p z f^{(p-1)}(z)-2 p f^{(p-2)}(z)\right|-\left|[(A-B)(2 p-\alpha)+2 p B] f^{(p-2)}(z)-p B f^{(p-1)}(z)\right| \\
& =\left|-\sum_{n=1^{\infty}} \frac{p n(p+n) !}{(n+2) !} a_{p+n} z^{n+2}\right| \\
& -\mid \frac{(A-B)(2 p-\alpha) p ! z^{2}}{2}-\left[\sum_{n=1} \frac{(A-B)(2 p-\alpha)(p+n) !}{(n+2) !} a_{p+n} z^{n+2}\right. \\
& \left.\leq \sum_{n=1}^{\infty} \frac{[p n(1-B)+(A-B)(2 p-\alpha)](p+n) !}{(n+2) !} \frac{p n(p+n) !}{(n+2) !} a_{p+n} z^{n+2}\right] \mid \\
& -\frac{1}{2}(A-B)(2 p-\alpha) p ! \leq 0
\end{aligned}
$$

by hypothesis. Hence, by the maximum modulus theorem, we have $f \in$ $A_{k}^{*}(p, A, B, \alpha)$.

To prove the converse, assume that

$$
\begin{gathered}
\left|\frac{p \frac{f^{(p-1)}(z)}{f^{(p-2)}(z)}-2 p}{(A-B)(2 p-\alpha)+2 p B-p B \frac{f^{(p-1)}(z)}{f^{(p-2)}(z)}}\right| \\
=\left|\frac{-2 \sum_{n=1}^{\infty} \frac{p n(p+n) !}{(n+2) !} a_{p+n} z^{n+2}}{(A-B)(2 p-\alpha) p ! z^{2}-\sum_{n=1}^{\infty} \frac{[(A-B)(2 p-\alpha)-B p n](p+n) !}{(n+2) !} a_{p+n} z^{n+2}}\right|<1 .
\end{gathered}
$$

Since $\operatorname{Re}(z) \leq|z|$ for all $z$, we have

$$
\operatorname{Re}\left\{\frac{-2 \sum_{n=1}^{\infty} \frac{p n(p+n) !}{(n+2) !} a_{p+n} z^{n+2}}{(A-B)(2 p-\alpha) p ! z^{2}-\sum_{n=1}^{\infty} \frac{[(A-B)(2 p-\alpha)-B p n](p+n) !}{(n+2) !} a_{p+n} z^{n+2}}\right\}<1 .
$$


Choose values of $z$ on the real axis so that $\frac{f^{(p-1)}(z)}{f^{(p-2)}(z)}$ is real. Upon clearing the denominator in (2.2) and letting $z \rightarrow 1^{-}$through real values, we obtain

$$
\sum_{n=1}^{\infty} \frac{[p n(1-B)+(A-B)(2 p-\alpha)](p+n) !}{(n+2) !} a_{p+n} \leq \frac{1}{2}(A-B)(2 p-\alpha) p ! .
$$

which obviously is required assertion (2.1).

Finally, sharpness follows if we take

$$
f(z)=z^{p}-\frac{(n+2) !(A-B)(p-\alpha) p !}{2[p n(1-B)+(A-B)(2 p-\alpha)](p+n) !} z^{p+n} ; n \geq 1 .
$$

Corollary 1 If $f \in A_{k}^{*}(p, A, B, \alpha)$, then

$$
a_{p+n} \leq \frac{(A-B)(2 p-\alpha)(n+2) ! p !}{2[p n(1-B)+(A-B)(2 p-\alpha)](p+n) !} .
$$

The equality in (2.5) is attained for the function $f$ given by (2.4).

\section{Distortion Properties}

Theorem 2 If $f \in A_{k}^{*}(p, A, B, \alpha)$, then for $|z|=r<1$

$$
\begin{gathered}
r^{p}-\frac{3(A-B)(2 p-\alpha)}{[p(1-B)+(A-B)(2 p-\alpha)](p+1)} r^{p+1} \leq|f(z)| \\
\leq r^{p}+\frac{3(A-B)(2 p-\alpha)}{[p(1-B)+(A-B)(2 p-\alpha)](p+1)} r^{p+1}
\end{gathered}
$$

and

$$
\begin{gathered}
p r^{p-1}-\frac{3(A-B)(2 p-\alpha)}{[p(1-B)+(A-B)(2 p-\alpha)]} r^{p} \leq\left|f^{\prime}(z)\right| \\
\leq p r^{p-1}+\frac{3(A-B)(2 p-\alpha)}{[p(1-B)+(A-B)(2 p-\alpha)]} r^{p} .
\end{gathered}
$$

All the inequalities are sharp.

Proof Let $f(z)=z^{p}-\sum_{n=1}^{\infty} a_{p+n} z^{p+n}$. From Theorem 1, we have

$$
\begin{aligned}
& \frac{[p(1-B)+(A-B)(2 p-\alpha)](p+1) !}{3 !} \sum_{n=1}^{\infty} a_{p+n} \\
& \leq \sum_{n=1}^{\infty} \frac{[p n(1-B)+(A-B)(2 p-\alpha)](p+n) !}{(n+2) !}
\end{aligned}
$$




$$
\leq \frac{1}{2}(A-B)(2 p-\alpha) p !
$$

which immediately yields for $n \geq 1$

$$
\begin{gathered}
\sum_{n=1}^{\infty} a_{p+n} \leq \frac{3(A-B)(2 p-\alpha)}{[p(1-B)+(A-B)(2 p-\alpha)](p+1)} \\
\sum_{n=1}^{\infty}(p+n) a_{p+n} \leq \frac{3(A-B)(2 p-\alpha)}{[p(1-B)+(A-B)(2 p-\alpha)]}
\end{gathered}
$$

Consequently, for $|z|=r<1$, we obtain

$$
\begin{aligned}
& |f(z)| \leq|z|^{p}+\sum_{n=1}^{\infty}\left|a_{p+n}\right||z|^{p+n} \leq r^{p}+r^{p+1} \sum_{n=1}^{\infty} a_{p+n} \\
& \leq r^{p}+\frac{3(A-B)(2 p-\alpha)}{[p(1-B)+(A-B)(2 p-\alpha)](p+1)} r^{p+1} \\
& |f(z)| \geq|z|^{p}-\sum_{n=1}^{\infty}\left|a_{p+n}\right||z|^{p+n} \geq r^{p}-r^{p+1} \sum_{n=1}^{\infty} a_{p+n} \\
& \geq r^{p}-\frac{3(A-B)(2 p-\alpha)}{[p(1-B)+(A-B)(2 p-\alpha)](p+1)} r^{p+1}
\end{aligned}
$$

which prove that the assertion(3.1) of Theorem 2. We also prove the inequalities in (3.2) as above. The bounds in (3.1) and (3.2) are attained for the function $f$ given by

$$
f(z)=z^{p}-\frac{3(A-B)(2 p-\alpha)}{[p(1-B)+(A-B)(2 p-\alpha)](p+1)} z^{p+1} .
$$

Letting $r \rightarrow 1^{-}$in the left hand side of (3.1), we have the following:

Corollary 2 If $f \in A_{k}^{*}(p, A, B, \alpha)$, then the disc $|z|<1$ is mapped by $f$ onto a domain that contains the disc

$$
|w|<\frac{p(1-B)(p+1)+(A-B)(2 p-\alpha)(p-2)}{[p(1-B)+(A-B)(2 p-\alpha)](p+1)} .
$$

The result is sharp with extremal function $f$ being given by (3.6)

Corollary 3 If $f \in A_{k}^{*}(p, A, B, 0)$, then for $|z|=r$

$$
r^{p}-\frac{6 p(A-B)}{[p(1-B)+(A-B)(2 p-\alpha)](p+1)} r^{p+1} \leq|f(z)|
$$




$$
\leq r^{p}+\frac{6 p(A-B)}{[p(1-B)+(A-B)(2 p-\alpha)](p+1)} r^{p+1}
$$

and

$$
\begin{gathered}
p r^{p-1}-\frac{6 p(A-B)}{[p(1-B)+(A-B)(2 p-\alpha)]} r^{p} \leq\left|f^{\prime}(z)\right| \\
\leq p r^{p-1}-\frac{6 p(A-B)}{[p(1-B)+(A-B)(2 p-\alpha)]} r^{p} .
\end{gathered}
$$

The result is sharp with the extremal function

$$
f(z)=z^{p}-\frac{6 p(A-B)}{[p(1-B)+(A-B)(2 p-\alpha)](p+1)]} z^{p+1} .
$$

Corollary 4 If $f \in A_{k}^{*}(p, A, B, 0)$, then the disc $|z|<1$ is mapped by $f$ onto a domain that contains the disc

$$
|w|<\frac{p(1-B)(p+1)+(A-B) 2 p(p-2)}{[p(1-B)+(A-B) 2 p](p+1)} .
$$

The result is sharp with extremal function $f$ being given by (3.7)

\section{Radii of Close-to-Convexity, Starlikeness and Convexity}

Theorem 3 Let the function $f$ be defined by (1.4) in the class $A_{k}^{*}(p, A, B, \alpha)$. Then $f$ is p-valent close-to-convex of order $\delta(0 \leq \delta \leq p)$ in $|z|<R_{1}$ where

$$
R_{1}=i n f_{n}\left\{\left[\frac{2[p n(1-B)+(A-B)(2 p-\alpha)](p+n) !}{(A-B)(2 p-\alpha) p !(n+2) !}\left(\frac{p-\delta}{p+n}\right)\right]^{\frac{1}{n}}\right\} .
$$

Theorem 4 If $f \in A_{k}^{*}(p, A, B, \alpha)$ then $f$ is p-valent starlike of order $\delta(0 \leq$ $\delta \leq p)$ in $|z|<R_{2}$ where

$$
R_{2}=i n f_{n}\left\{\left[\frac{2[p n(1-B)+(A-B)(2 p-\alpha)](p+n) !}{(A-B)(2 p-\alpha) p !(n+2) !}\left(\frac{p-\delta}{p+n-\delta}\right)\right]^{\frac{1}{n}}\right\} .
$$

Theorem 5 If $f \in A_{k}^{*}(p, A, B, \alpha)$ then $f$ is p-valent convex of order $\delta(0 \leq$ $\delta \leq p)$ in $|z|<R_{3}$ where

$$
R_{3}=\inf _{n}\left\{\left[\frac{2[p n(1-B)+(A-B)(2 p-\alpha)](p+n-1) !}{(A-B)(2 p-\alpha) p !(n+2) !}\left(\frac{p(p-\delta)}{p+n-\delta}\right)\right]^{\frac{1}{n}}\right\}
$$

In order to establish the required results in Theorem 3,4 and 5, it is sufficient to show that $\left|\frac{f^{\prime}(z)}{z^{p-1}}-p\right| \leq p-\delta$ for $|z|<R_{1},\left|\frac{z f^{\prime}(z)}{f(z)}-p\right| \leq p-\delta$ for $|z|<R_{2}$ 
and $\left|\left[1+\frac{z f^{\prime \prime}(z)}{f^{\prime}(z)}\right]-p\right| \leq p-\delta$ for $|z|<R_{3}$, respectively.

Remark The results in Theorem 3,4 and 5 are sharp with extremal function $f$ given by (2.4). Furthermore, taking $\delta=0$ in Theorem 3,4 and 5, we obtain radii of close-to-convexity, starlikeness and convexity, respectively.

\section{Integral Operators}

Theorem 6 Let $c$ be real number such that $c>-p$. If $f \in A_{k}^{*}(p, A, B, \alpha)$ then the function $F$ defined by

$$
f(z)=\frac{c+p}{z^{c}} \int_{0}^{z} t^{c-1} f(t) d t
$$

also belongs to $A_{k}^{*}(p, A, B, \alpha)$.

Proof Let $f(z)=z^{p}-\sum_{n=1}^{\infty} a_{p+n} z^{p+n}$. Then from representation of $F$, it follows that

$$
F(z)=z^{p}-\sum_{n=1}^{\infty} b_{p+n} z^{p+n}
$$

where

$$
b_{p+n}=\left(\frac{c+p}{c+p+n}\right) a_{p+n} .
$$

Therefore using Theorem 1 for the coefficients of $F$ we have

$$
\begin{aligned}
& \sum_{n=1}^{\infty} \frac{[p n(1-B)+(A-B)(2 p-\alpha)](p+n) !}{(n+2) !} b_{p+n} \\
= & \sum_{n=1}^{\infty} \frac{[p n(1-B)+(A-B)(2 p-\alpha)](p+n) !}{(n+2) !}\left(\frac{c+p}{c+p+n}\right) a_{p+n} \\
\leq & \frac{1}{2}(A-B)(2 p-\alpha) p !
\end{aligned}
$$

since $\frac{c+p}{c+p+n}<1$ and $f \in A_{k}^{*}(p, A, B, \alpha)$. Hence $F \in A_{k}^{*}(p, A, B, \alpha)$.

Theorem 7 Let $c$ be real number such that $c>-p$. If $F \in A_{k}^{*}(p, A, B, \alpha)$, then the function $f(z)$ defined by (5.1) is p-valent in $|z|<R^{*}$, where

$$
R^{*}=i n f_{n}\left\{\left[\left(\frac{c+p}{c+p+n}\right) \frac{2[p n(1-B)+(A-B)(2 p-\alpha)](p+n-1) !}{(n+2) !(A-B)(2 p-\alpha)(p-1) !}\right]^{\frac{1}{n}}\right\}
$$


The result is sharp.

Sharpness follows if we take

$$
f(z)=z^{p}-\left(\frac{c+p+n}{c+p}\right) \frac{(n+2) !(A-B)(2 p-\alpha) p !}{2[p n(1-B)+(A-B)(2 p-\alpha)](p+n) !} z^{p+n} .
$$

\section{Closure Properties}

In this section we show that the class $A_{k}^{*}(p, A, B, \alpha)$ is closed under "arithmetic mean" and "convex linear combinations".

Theorem 8 Let $f_{j}(z)=z^{p}-\sum_{n=1}^{\infty} a_{p+n, j} z^{p+n} ; j=1,2, \ldots$ and $h(z)=$ $z^{p}-\sum_{n=1}^{\infty} b_{p+n} z^{p+n}$ where $b_{p+n}=\sum_{n=1}^{\infty} \lambda_{j} a_{p+n, j}, \lambda_{j}>0$ and $\sum_{n=1}^{\infty} \lambda_{j}=1$ If $f_{j} \in A_{k}^{*}(p, A, B, \alpha)$ for each $j=1,2, \ldots$, then $h \in A_{k}^{*}(p, A, B, \alpha)$.

Proof If $f_{j} \in A_{k}^{*}(p, A, B, \alpha)$, then we have from Theorem 1 that

$$
\begin{gathered}
\sum_{n=1}^{\infty} \frac{[p n(1-B)+(A-B)(2 p-\alpha)](p+n) !}{(n+2) !} a_{p+n, j} \\
\leq \frac{1}{2}(A-B)(2 p-\alpha) p ! ; j=1,2, \ldots
\end{gathered}
$$

Therefore

$$
\begin{aligned}
& \sum_{n=1}^{\infty} \frac{[p n(1-B)+(A-B)(2 p-\alpha)](p+n) !}{(n+2) !} b_{p+n} \\
= & \sum_{n=1}^{\infty}\left\{\frac{[p n(1-B)+(A-B)(2 p-\alpha)](p+n) !}{(n+2) !}\left(\sum_{n=1}^{\infty} \lambda_{j} a_{p+n, j}\right)\right\} \\
\leq & \frac{1}{2}(A-B)(2 p-\alpha) p ! .
\end{aligned}
$$

Hence, by Theorem $1, h \in A_{k}^{*}(p, A, B, \alpha)$.

Theorem 9 The class $A_{k}^{*}(p, A, B, \alpha)$ is closed under convex linear combinations.

Theorem 10 Let $f_{p}(z)=z^{p}$ and 
$f_{p+n}=z^{p}-\frac{(A-B)(2 p-\alpha)(n+2) ! p !}{2[p n(1-B)+(A-B)(2 p-\alpha)](p+n) !} z^{p+n},(n \geq 1)$. Then $f \in A_{k}^{*}(p, A, B, \alpha)$ if and only if it can be expressed in the form

$$
f(z)=\lambda_{p} f_{p}(z)+\sum_{n=1}^{\infty} \lambda_{n} f_{p+n}(z), z \in \mathbb{U},
$$

where $\lambda_{n} \geq 0$ and $\lambda_{p}=1-\sum_{n=1}^{\infty} \lambda_{n}$.

Proof Let us that

$$
\begin{gathered}
f(z)=\lambda_{p} f_{p}(z)+\sum_{n=1}^{\infty} \lambda_{n} f_{p+n}(z) \\
=\left[1-\sum_{n=1}^{\infty} \lambda_{n}\right] z^{p}+\sum_{n=1}^{\infty} \lambda_{n}\left\{z^{p}-\frac{(A-B)(2 p-\alpha)(n+2) ! p !}{2[p n(1-B)+(A-B)(2 p-\alpha)](p+n) !} z^{p+n}\right\} \\
=z^{p}-\sum_{n=1}^{\infty} \frac{(A-B)(2 p-\alpha)(n+2) ! p !}{2[p n(1-B)+(A-B)(2 p-\alpha)](p+n) !} \lambda_{n} z^{p+n} .
\end{gathered}
$$

Then from Theorem 1, we have

$$
\begin{gathered}
\sum_{n=1}^{\infty} \frac{[p n(1-B)+(A-B)(2 p-\alpha)](p+n) !}{(n+2) !} \frac{(A-B)(2 p-\alpha)(n+2) ! p !}{2[p n(1-B)+(A-B)(2 p-\alpha)](p+n) !} \lambda_{n} \\
\leq \frac{1}{2}(A-B)(2 p-\alpha) p ! \sum_{n=1}^{\infty} \lambda_{n} \leq \frac{1}{2}(A-B)(2 p-\alpha) p ! .
\end{gathered}
$$

Hence $f \in A_{k}^{*}(p, A, B, \alpha)$. Conversely, let $f \in A_{k}^{*}(p, A, B, \alpha)$. Using Corollary 1 and setting $\lambda_{n}=\frac{2[p n(1-B)+(A-B)(2 p-\alpha)](p+n) !}{(A-B)(2 p-\alpha) p !(n+2) !} a_{p+n}, n=1,2, \ldots$ and $\lambda_{p}=1-$ $\sum_{n=1}^{\infty} \lambda_{n}$, we have

$$
\begin{gathered}
f(z)=z^{p}-\sum_{n=1}^{\infty} a_{p+n} z^{p+n} \\
=z^{p}-\sum_{n=1}^{\infty} \lambda_{n} z^{p}-\sum_{n=1}^{\infty} \lambda_{n} \frac{(A-B)(2 p-\alpha)(n+2) ! p !}{2[p n(1-B)+(A-B)(2 p-\alpha)](p+n) !} z^{p+n} \\
=\lambda_{p} f_{p}(z)+\sum_{n=1}^{\infty} \lambda_{n} f_{p+n}(z) .
\end{gathered}
$$

This completes the proof of Theorem 10 . 


\section{Definitions and Applications of the Fractional Calculus}

In this section, we shall prove several distortion theorems for functions to general class $A_{k}^{*}(p, A, B, \alpha)$. Each of these theorems would involve certain operators of fractional calculus we find it to be convenient to recall here the following definition which were used recently by Owa [6] (and more recently, by Owa and Srivastava [7], and Srivastava and Owa [2], ; see also Srivastava et all. [1] )

Definition 1 The fractional integral of order $\lambda$ is defined, for a function $f$, by

$$
D_{z}^{-\lambda} f(z)=\frac{1}{\Gamma(\lambda)} \int_{0}^{z} \frac{f(\xi)}{(z-\xi)^{1-\lambda}} d \xi \quad ;(\lambda>0)
$$

where $f$ is an analytic function in a simply - connected region of the $z$-plane containing the origin, and the multiplicity of $(z-\xi)^{\lambda-1}$ is removed by requiring $\log (z-\xi)$ to be real when $z-\xi>0$.

Definition 2 The fractional derivative of order $\lambda$ is defined, for a function $f$, by

$$
D_{z}^{\lambda} f(z)=\frac{1}{\Gamma(1-\lambda)} \frac{d}{d z} \int_{0}^{z} \frac{f(\xi)}{(z-\xi)^{\lambda}} d \xi ;(0 \leq \lambda<1)
$$

where $f$ is constrained, and the multiplicity of $(z-\xi)^{-\lambda}$ is removed, as in Definition 1.

We remark that in Definition 1 and 2, $\Gamma$ denotes the gamma function.

Definition 3 Under the hypotheses of Definition 2, the fractional derivative of order $(n+\lambda)$ is defined by

$$
D_{z}^{n+\lambda} f(z)=\frac{d^{n}}{d z^{n}} D_{z}^{\lambda} f(z)
$$

where $0 \leq \lambda<1$ and $n \in \mathbb{N}_{0}=\mathbb{N} \cup\{0\}$. From Definition 2, we have

$$
D_{z}^{0} f(z)=f(z)
$$

which, inview of Definition 3 yields,

$$
D_{z}^{n+0} f(z)=\frac{d^{n}}{d z^{n}} D_{z}^{0} f(z)=f^{n}(z) .
$$

Thus, it follows from (8.4) and (8.5) that $\lim _{\lambda \rightarrow 0} D_{z}^{-\lambda} f(z)=f(z)$ and $\lim _{\lambda \rightarrow 0} D_{z}^{1-\lambda} f(z)=f^{\prime}(z)$. 
Theorem 11 Let the function $f$ defined by (1.4) be in the class $A_{k}^{*}(p, A, B, \alpha)$. Then for $z \in \mathbb{U}$ and $\lambda>0$,

$$
\left|D_{z}^{-\lambda} f(z)\right| \geq \frac{\Gamma(p+1)}{\Gamma(\lambda+p+1)} z^{p+\lambda}\left\{1-\frac{3(A-B)(2 p-\alpha)}{[p(1-B)+(A-B)(2 p-\alpha)](p+1)}|z|\right\}
$$

and

$$
\left|D_{z}^{-\lambda} f(z)\right| \leq \frac{\Gamma(p+1)}{\Gamma(\lambda+p+1)} z^{p+\lambda}\left\{1+\frac{3(A-B)(2 p-\alpha)}{[p(1-B)+(A-B)(2 p-\alpha)](p+1)}|z|\right\} .
$$

The result is sharp.

Proof Let

$$
\begin{gathered}
F(z)=\frac{\Gamma(p+1+\lambda)}{\Gamma(p+1)} z^{-\lambda} D_{z}^{-\lambda} f(z) \\
=z^{p}-\sum_{n=1}^{\infty} \frac{\Gamma(p+n+1) \Gamma(p+\lambda+1)}{\Gamma(p+1) \Gamma(p+n+\lambda+1)} a_{p+n} z^{p+n}=z^{p}-\sum_{n=1}^{\infty} A_{p+n} z^{p+n}
\end{gathered}
$$

where

$$
A_{p+n}=\frac{\Gamma(p+n+1) \Gamma(p+\lambda+1)}{\Gamma(p+1) \Gamma(p+n+\lambda+1)} a_{p+n} .
$$

Then by using $0<A_{p+n} \leq a_{p+n}$ and Theorem 1 , we observe that

$$
\begin{aligned}
& \sum_{n=1}^{\infty} \frac{[p n(1-B)+(A-B)(2 p-\alpha)](p+n) !}{(n+2) !} A_{p+n} \\
& \leq \sum_{n=1}^{\infty} \frac{[p n(1-B)+(A-B)(2 p-\alpha)](p+n) !}{(n+2) !} a_{p+n} \\
& \leq \frac{1}{2}(A-B)(2 p-\alpha) p !
\end{aligned}
$$

which shows that $F \in A_{k}^{*}(p, A, B, \alpha)$. Consequently, with aid of Theorem 2, we have

$$
|F(z)| \geq z^{p}-\frac{3(A-B)(2 p-\alpha)}{[p(1-B)+(A-B)(2 p-\alpha)](p+1)}|z|^{p+1}
$$


and

$$
|F(z)| \leq z^{p}+\frac{3(A-B)(2 p-\alpha)}{[p(1-B)+(A-B)(2 p-\alpha)](p+1)}|z|^{p+1}
$$

which completes the proof of Theorem 11. By letting $\lambda \rightarrow 0$, Theorem 11 reduces at once to Theorem 2 .

Corollary 5 Under the hypotheses of Theorem 11, $D_{z}^{-\lambda} f(z)$ is included in a disk with its center at the origin and radius $R_{1}^{-\lambda}$ given by

$$
R_{1}^{-\lambda}=\left\{\frac{\Gamma(p+1)}{\Gamma(\lambda+p+1)}\right\}\left\{1+\frac{3(A-B)(2 p-\alpha)}{[p(1-B)+(A-B)(2 p-\alpha)](p+1)}\right\} .
$$

Theorem 12 Let the function $f$ defined by (1.4) be in the class $A_{k}^{*}(p, A, B, \alpha)$. Then,

$\left|D_{z}^{\lambda} f(z)\right| \geq \frac{\Gamma(p+1)}{\Gamma(p+1-\lambda)} z^{p-\lambda}\left\{1-\frac{3(A-B)(2 p-\alpha)}{[p(1-B)+(A-B)(2 p-\alpha)](p-\lambda+1)}|z|\right\}$

and

$\left|D_{z}^{\lambda} f(z)\right| \leq \frac{\Gamma(p+1)}{\Gamma(p+1-\lambda)} z^{p+\lambda}\left\{1+\frac{3(A-B)(2 p-\alpha)}{[p(1-B)+(A-B)(2 p-\alpha)](p-\lambda+1)}|z|\right\}$.

for $0 \leq \lambda<1$.

Proof Using similar arguments as given by Theorem 11, we can get result.

Corollary 6 Under the hypotheses of Theorem $12, D_{z}^{\lambda} f(z)$ is included in a disk with its center at the origin and radius $R_{2}^{\lambda}$ given by

$$
R_{2}^{\lambda}=\left\{\frac{\Gamma(p+1)}{\Gamma(p+1-\lambda)}\right\}\left\{1+\frac{3(A-B)(2 p-\alpha)}{[p(1-B)+(A-B)(2 p-\alpha)](p+1-\lambda)}\right\} .
$$

\section{References}

[1] H.M.SRIVASTAVA, M.SAIGO and S.OWA, A class of distortion theorems involving certain operators of fractional calculus, J.Math.Anal.Appl.131(1988 ), 412-420.

[2] H.M.SRIVASTAVA and S.OWA, Some Characterization and distortion theorems involving fractional calculus, linear operators and certain subclasses of analytic functions,Nagoya Math.J.106 (1987),1-28. 
[3] M. NUNOKAWA and S. HOSHINO, On some starlikeness conditions for analytic functions, Tsukuba J. Math. Vol.16 No.1 (1992), 211-215.

[4] M.K. AOUF and H.E. DARWISH, Basic Properties and characterizations of a certain class of Analytic funtions with negative coefficients II, Utilitas Math.46 (1994), 167-177.

[5] S.L.SHUKLA and DASTRATH, On certain classes of multivalent functions with negative coefficients, Pure and Appl. Math.Sci. 20(1984), 1-2, 63-72.

[6] S.OWA, On the distortion theorems,I.Kyunpook Math.J. 18 ( 1978 ), 5359.

[7] S.OWA and H.M.SRIVASTAVA, Univalent and Starlike generalized hypergeometric functions, Canad. J. Math. 39 (1987 ), 1057-1077.

\section{Received: October 3, 2005}

